Case Report

\title{
Inferior Right Hepatic Vein: A Useful Anatomic Variation for Isolated Resection of Segment VIII
}

\author{
Klaus Steinbrück, Reinaldo Fernandes, Giuliano Bento, Rafael Vasconcelos, \\ Gustavo Stoduto, Thomas Auel, and Lúcio F. Pacheco-Moreira \\ Hepatobiliary Surgery Unit, Bonsucesso Federal Hospital-Health Ministry, Av. Londres, 616, prédio 3/2 ${ }^{\circ}$ andar, \\ 21041-030 Rio de Janeiro, RJ, Brazil \\ Correspondence should be addressed to Klaus Steinbrück; drsteinbruck@yahoo.com.br
}

Received 24 June 2013; Accepted 24 July 2013

Academic Editors: F. Catena, T. Çolak, and Y.-B. Tang

Copyright (C) 2013 Klaus Steinbrück et al. This is an open access article distributed under the Creative Commons Attribution License, which permits unrestricted use, distribution, and reproduction in any medium, provided the original work is properly cited.

Anatomical resection of segment VIII (SVIII) is one of the most difficult hepatectomies to perform. Although it is the best choice of surgical treatment for tumors located at SVIII, its feasibility can be compromised when the right hepatic vein (RHV) must be resected en bloc with SVIII. Herein we describe a case of a cirrhotic patient that was submitted to segmentectomy VIII in bloc with the main trunk of the RHV, due to hepatocellular carcinoma. The resection could only be performed because a well developed inferior right hepatic vein (IRHV) was present. Anatomical variations of the liver vascularization should be used by liver surgeons to improve surgical results.

\section{Background}

Right hepatectomy is the procedure of choice for most patients with normal liver function and hepatic tumors located in the right liver, especially those in close contact to the right hepatic vein (RHV). Parenchyma-sparing resection may be required, however, in patients with impaired liver function and this is common in patients with cirrhosis and hepatocellular carcinoma (HCC).

For tumors confined to segment VIII (SVIII) of the liver, the procedure of segmentectomy VIII offers a chance of anatomically resecting the tumor while preserving most of the liver tissue. This procedure, however, can be really challenging when the tumor is to close to the RHV.

Up to $21 \%$ of patients have a large inferior right hepatic vein (IRHV) that drains the inferior segments of the right hemiliver [1]. The presence of this variation facilitates the isolated resection of SVIII, even when the RHV must be ligated and divided.

Herein we describe a case of isolated segmentectomy VIII due to HCC in a cirrhotic patient, in which the presence of an IRHV enabled the section of the RHV without harming the drainage of inferior segments.

\section{Patient and Methods}

A 66-year-old woman was admitted to our hospital with an abdominal ultrasonography showing a $2 \mathrm{~cm}$ nodule in SVIII of the liver. She had been previously diagnosed with Hepatitis $\mathrm{B}$, but received no further treatment. Laboratory tests revealed AST $34 \mathrm{U} / \mathrm{L}$, ALT $28 \mathrm{U} / \mathrm{L}$, total bilirubin $0.8 \mathrm{mg} / \mathrm{dL}$, INR 1.1 and albumin $4.5 \mathrm{~g} / \mathrm{dL}$, which demonstrated a minor liver disfunction. As she had no ascites or encephalopathy, she was classified as Child-Pugh's grade A. Alpha-fetoprotein level was $845 \mathrm{ng} / \mathrm{mL}$. MRI confirmed a 2.5 tumor touching and compressing the main trunk of RHV (Figure 1) and was consistent with HCC. The exam revealed also an IRHV (Figure 2) draining segments V and VI directly into the inferior vena cava, which encourage us to perform an isolated resection of segment VIII with ligation and division of the RHV.

Surgery was performed by bilateral subcostal laparotomy. Structures of the hepatic pedicle were dissected. Right portal vein (RPV) and right hepatic artery (RHA) were isolated. Retrohepatic inferior vena cava was dissected. RHV and IRHV were encircled (Figure 3). Using intraoperative ultrasonography, the tumor and portal branches for SVIII were 


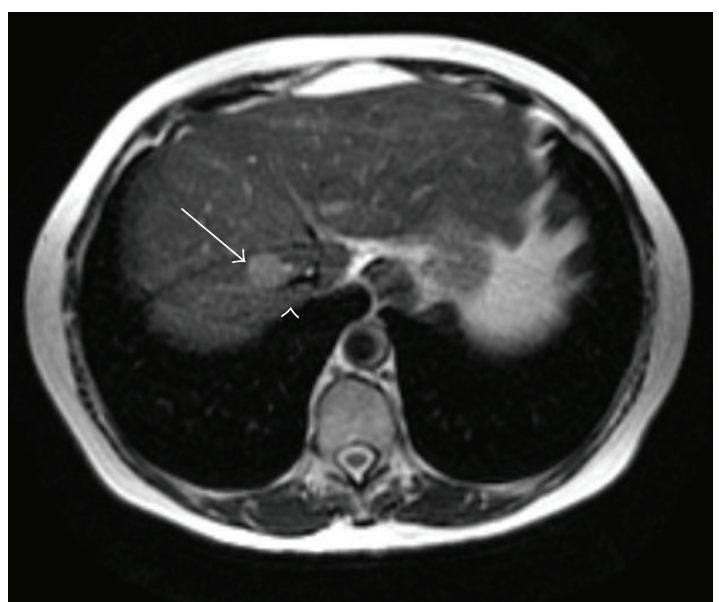

FIGURE 1: T2 weighted Magnetic Resonance image showing a tumor (arrow) compressing the RHV (arrow head).

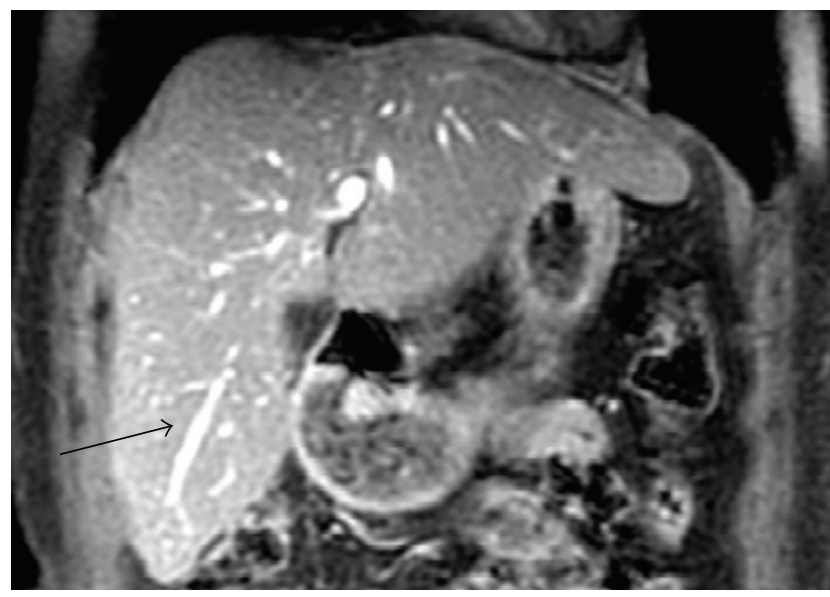

FIGURE 2: T1 weighted Magnetic Resonance image showing inferior right hepatic vein (arrow).

identified and resection area was marked in the liver surface. Segmentectomy VIII was performed with kellyclasia after occlusion of RPV and RHA for 26 minutes $(2 \times 10$ minutes and $1 \times 6$ minutes clamping with 10 minutes interval between them). The main trunk of the RHV was ligated and divided in the beginning of the procedure. Control of biliary leakage was obtained with injection of methylene blue through the cystic duct after cholecystectomy. Final aspect of surgery is shown in Figure 4.

On post-operative $(\mathrm{PO})$ period, AST, ALT, total bilirubin and INR raised up to $288 \mathrm{U} / \mathrm{L}, 254 \mathrm{U} / \mathrm{L}, 0.7 \mathrm{mg} / \mathrm{dL}$ and 1.62 , respectively. On PO day 6, patient presented fever and abdominal pain. CT scan revealed fluid collection at right subphrenic space, which was confirmed as a biloma after percutaneous drainage. Patient was discharge on PO day 16 after 10 days of antibiotic therapy. She had no further complications and is doing well, with no signs of HCC relapse, after 18 months.

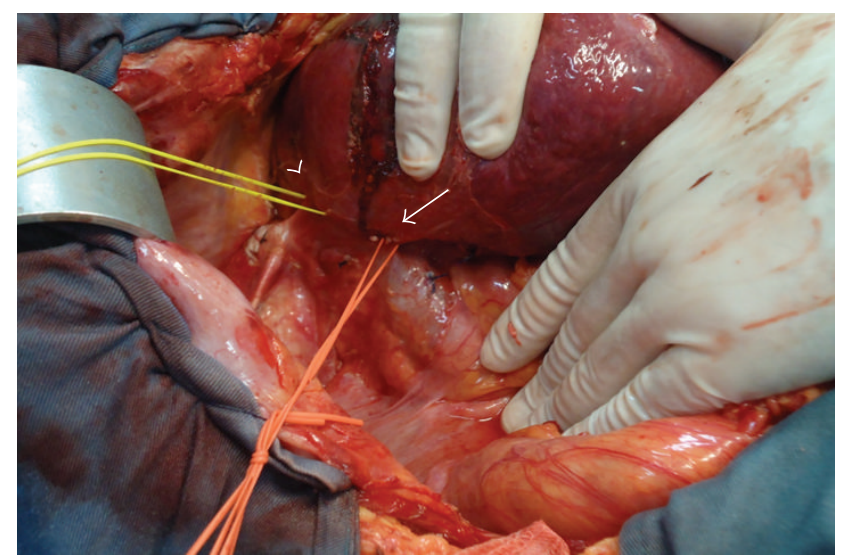

FIGURE 3: Retrohepatic inferior vena cava. RHV (arrow head) and inferior right hepatic vein (arrow) are encircled.

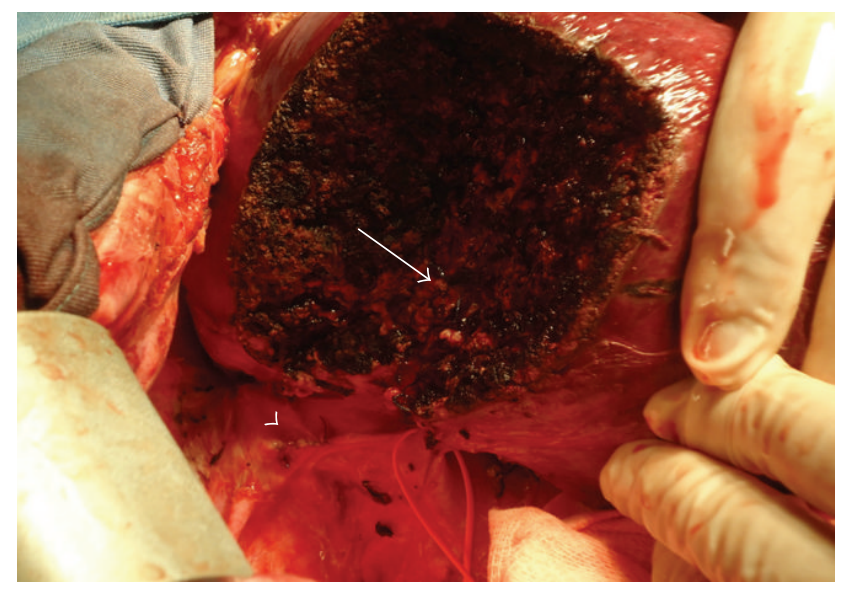

FIgURE 4: Final aspect of surgery. Divided RHV (arrow head) and portal pedicle (arrow) can be seen.

\section{Discussion}

Patients with large or deeply located liver tumors in SVIII are normally submitted to right hepatectomy or extended right hepatectomy to guarantee an oncological resection of the tumor. However, these major resections may not be tolerated by patients with poor liver reserve, which is often seen in HCC associated to cirrhosis. As HCC tends to metastasize via the portal vein [2], resection of the liver parenchyma fed by portal venous branches bearing the tumour seems logical to eradicate potential intrahepatic metastases. Therefore, isolated segmentectomy can be performed to achieve both oncological and parenchyma-sparing resection.

Among segmentectomies, isolated resection of SVIII is the most challenging and complex liver resections to perform. This segment is surrounded by the RHV laterally, the middle hepatic vein medially, and the anterior branch of the RPV inferiorly. Moreover, the absence of anatomic landmarks on the liver surface makes it difficult to determine the precise extent of resection. All these circumstances render an isolated resection of SVIII technically demanding, but still feasible to 
be done. Surgical techniques have already been described to provide an anatomical and secure resection of SVIII [3-5].

In particular cases, as the one described here, the tumor may be in close contact to the RHV and its resection together with the tumor is necessary. For these cases, drainage of the right liver can be seriously compromised, unless a variant drainage system of the hepatic veins is present. Fang et al. [1] reported a study of variations of the hepatic veins and showed that in $39 \%$ of patients, RHV is not the only responsible for entire drainage of right hemiliver. A well-developed IRHV is presented in $21 \%$ of cases and provides drainage for segments $\mathrm{V}$ and VI.

In the case herein reported, we performed the segmentectomy VIII associated to RHV resection because a IRHV was identified. If such anatomical variation was not present, this patient would have been elected for liver transplant, a much more aggressive treatment.

Machado et al. [6] and Capussotti et al. [7], both in 2006, described 5 cases that resection of segments VII-VIII and RHV was successfully performed in patients without an IRHV. However, for the best of our knowledge, since 2006 only eight more similar cases were reported [8] and no cases of isolated resection of SVIII and RHV without an IRHV were described until now. We believe that the presence of an IRHV is still a limitation for segmentectomy VIII with RHV ligation and division.

In conclusion, every patient eligible for a liver surgery must have his liver anatomy evaluated. Liver surgeons should be prepared to use anatomy variations in favor of their patients. Inferior right hepatic vein is a useful variation for isolated resection of SVIII, when the RHV must be sacrificed.

\section{Conflict of Interests}

There are no conflicts.

\section{Supportive Foundations}

There was no funding source.

\section{References}

[1] C. Fang, J. You, W. Y. Lau et al., "Anatomical variations of hepatic veins: three-dimensional computed tomography scans of 200 subjects," World Journal of Surgery, vol. 36, pp. 120-124, 2012.

[2] T. Nakashima and M. Kojiro, "Pathologic characteristics of hepatocellular carcinoma," Seminars in Liver Disease, vol. 6, no. 3, pp. 259-266, 1986.

[3] A. Mazziotti, A. Maeda, G. Ercolani, M. Cescon, G. L. Grazi, and F. Pierangeli, "Isolated resection of segment 8 for liver tumors: a new approach for anatomical segmentectomy," Archives of Surgery, vol. 135, no. 10, pp. 1224-1229, 2000.

[4] G. Torzilli, F. Procopio, A. Palmisano et al., "Total or partial anatomical resection of segment 8 using the ultrasound-guided finger compression technique," $H P B$, vol. 13, no. 8, pp. 586-591, 2011.

[5] J. Hu, W. Dai, X. Miao et al., "Anatomic resection of segment VIII of liver for hepatocellular carcinoma in cirrhotic patients based on an intrahepatic Glissonian approach," Surgery, vol. 146, no. 5, pp. 854-860, 2009.

[6] M. A. C. Machado, P. Herman, F. F. Makdissi, E. R. R. Figueira, T. Bacchella, and M. C. C. Machado, "Feasibility of bisegmentectomy 7-8 is independent of the presence of a large inferior right hepatic vein," Journal of surgical oncology, vol. 93, no. 4, pp. 338-342, 2006.

[7] L. Capussotti, A. Ferrero, L. Viganò, R. Polastri, D. Ribero, and E. Berrino, "Hepatic bisegmentectomy 7-8 for a colorectal metastasis," European Journal of Surgical Oncology, vol. 32, no. 4, pp. 469-471, 2006.

[8] J. S. Huang, W. D. Dai, X. Y. Miao et al., "Null-margin bisegmentectomy VII-VIII for hepatocellular carcinoma in cirrhotic patients," Hepatogastroenterology, vol. 59, pp. 1706-1709, 2012. 


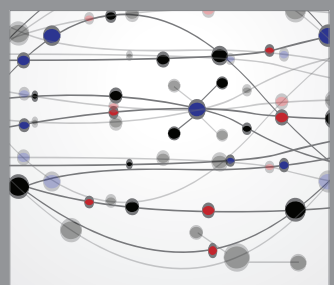

The Scientific World Journal
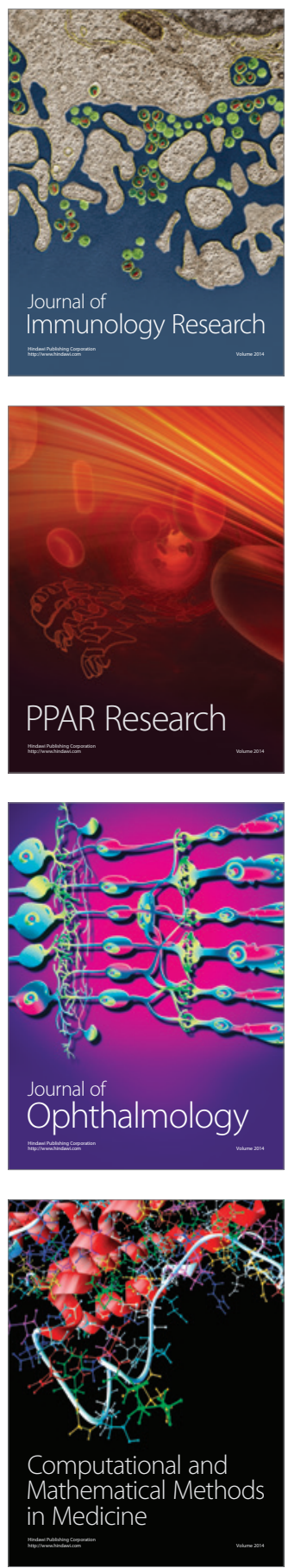

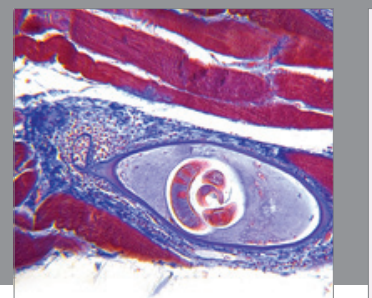

Gastroenterology

Research and Practice
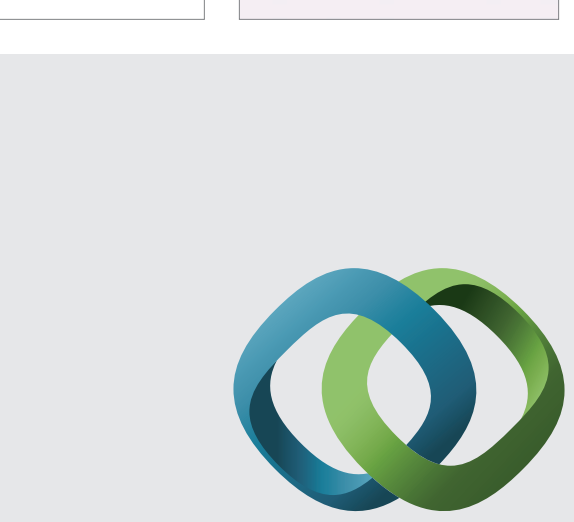

\section{Hindawi}

Submit your manuscripts at

http://www.hindawi.com
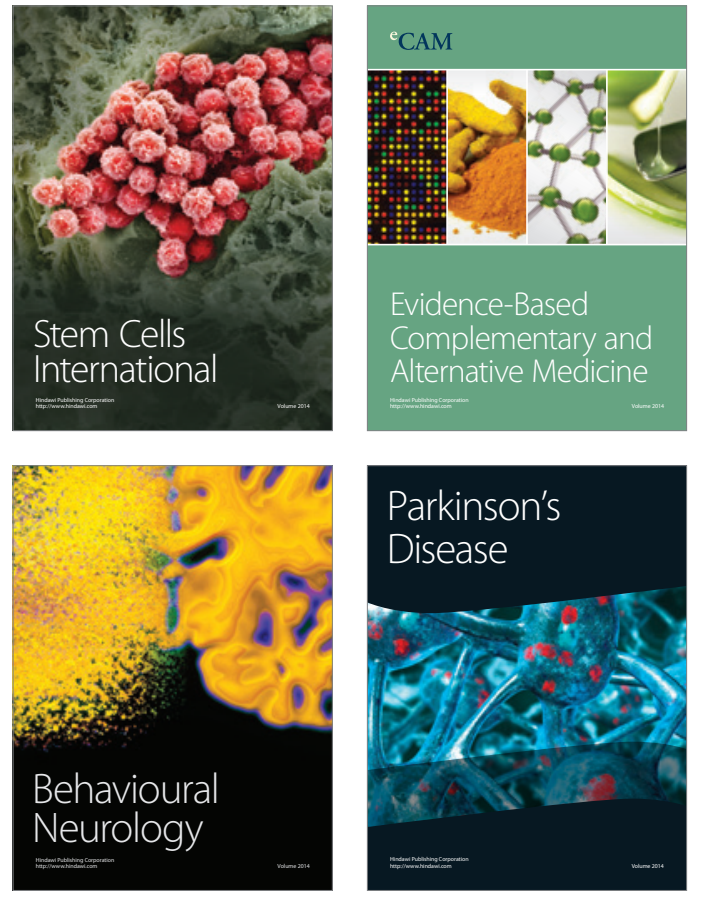
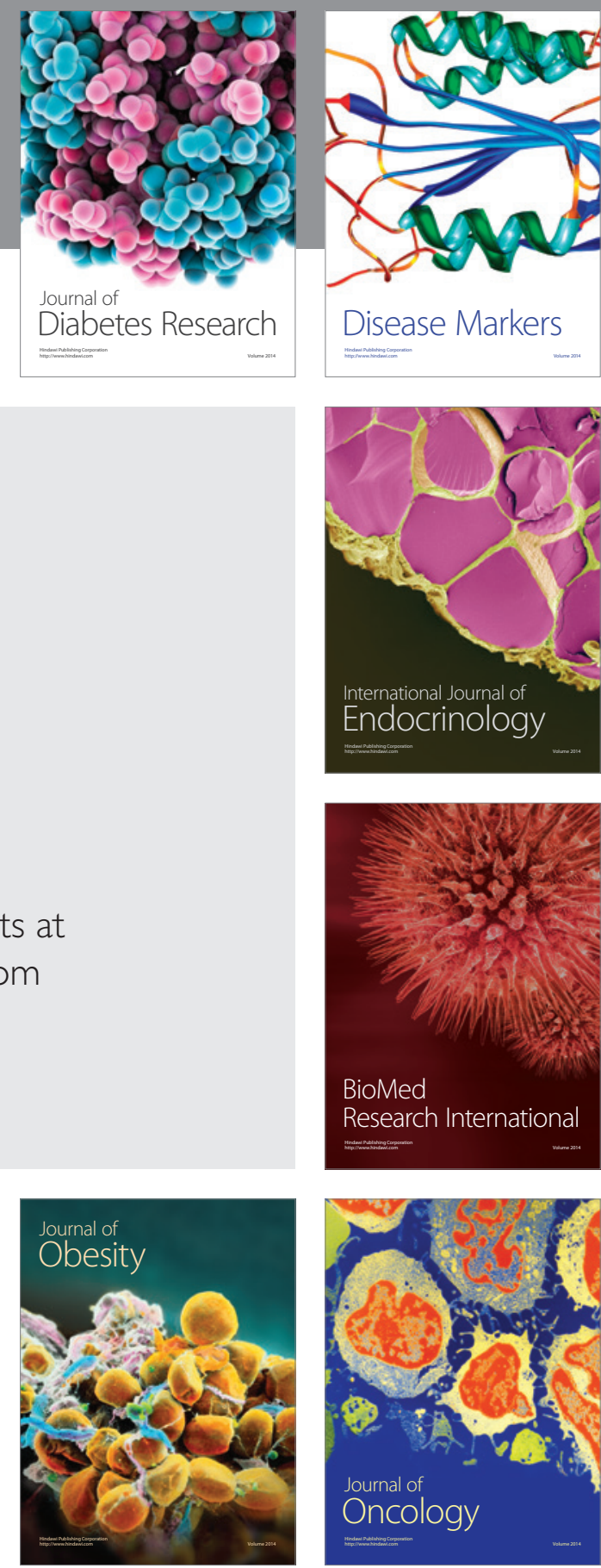

Disease Markers
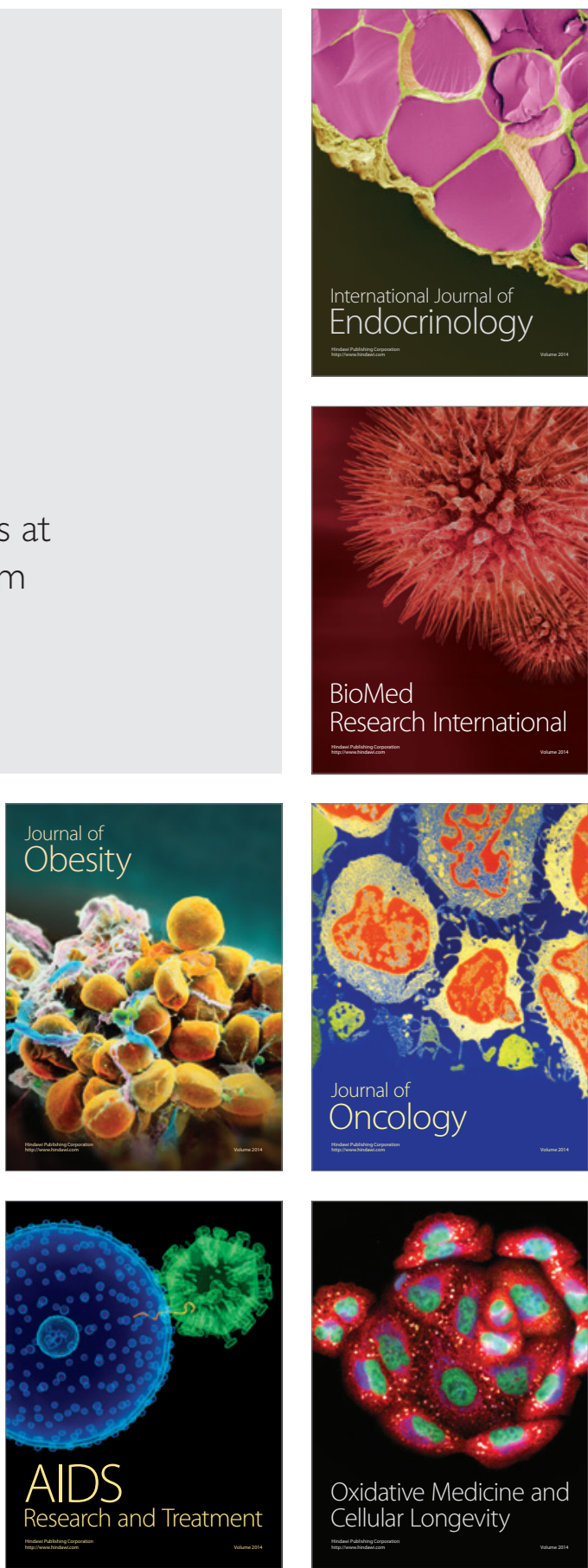